\title{
Editorial
}

\section{Nonlinear Problems: Analytical and Computational Approach with Applications}

\author{
Khalida Inayat Noor, ${ }^{1}$ Muhammad Aslam Noor, ${ }^{1}$ \\ Syed Tauseef Mohyud-Din, ${ }^{2}$ Ahmet Yildirim, ${ }^{3}$ \\ and Shaher M. Momani ${ }^{4}$ \\ ${ }^{1}$ Department of Mathematics, COMSATS Institute of Information Technology, Park Road, \\ Islamabad, Pakistan \\ ${ }^{2}$ Department of Mathematics, HITEC University, Taxila Cantt., Islamabad, Pakistan \\ ${ }^{3}$ Department of Mathematics, Ege University, Bornova, 35100 Izmir, Turkey \\ ${ }^{4}$ Department of Mathematics, Faculty of Science, The University of Jordan, Amman 11942, Jordan \\ Correspondence should be addressed to Khalida Inayat Noor, khalidanoor@hotmail.com
}

Received 15 August 2012; Accepted 15 August 2012

Copyright (C) 2012 Khalida Inayat Noor et al. This is an open access article distributed under the Creative Commons Attribution License, which permits unrestricted use, distribution, and reproduction in any medium, provided the original work is properly cited.

The nonlinear sciences have been taking new dimensions with the passage of time. The thorough study of the literature witnesses the introduction of a number of new techniques which are being used to cope with the versatility of diversified physical problems, and in the same context, a number of numerical and analytical techniques have been developed to cope with the physical nature of such equations. The basic purpose of this special issue is to extend the applications of the relatively new approaches and theories for nonlinear problems of physical nature arising in mathematics, physics, and applied sciences and to see the latest developments in the same context. The authors were invited to submit original research articles as well as review articles that stimulated the continuing efforts in nonlinear equations and related theories. The special issue provided a forum for researches and scientists to communicate their recent developments and to present their novel results on nonlinear equations and related problems. The topics included in this special issue are solitary wave theory, solitons and compactons, geometric function theory and its applications, symmetries and separation of variables, Hirota's bilinear method, Painlevé test and analysis, HamiltonJacobi approach, variational inequalities and their applications, analytical methods such as variational iteration techniques and its various modifications, variation of parameters method, continuous and discrete integrable equations, numerical approaches to nolinear waves, and finally stability theory of solitary waves.

(i) Solitary wave theory.

(ii) Solitons and compactons. 
(iii) Geometric function theory and its applications.

(iv) Symmetries and separation of variables.

(v) Hirota's bilinear method.

(vi) Painlevé test and analysis.

(vii) Hamilton-Jacobi approach.

(viii) Variational inequalities and their applications.

(ix) Analytical methods such as variational iteration techniques and its various Modifications.

(x) Variation of parameters method.

(xi) Continuous and discrete integrable equations.

(xii) Numerical approaches to nonlinear waves.

(xiii) Stability theory of solitary waves.

Keeping in view the recent trends in nonlinear problems: Abstract and Applied Analysis was selected: "Nonlinear Problems: Analytical and Computational Approach with Applications," as the theme of its special issue. The response to this special issue was beyond our expectation. We received 251 papers in the interdisciplinary research fields. This special issue includes 77 high-quality peer-reviewed articles that deal with different aspects of nonlinear problems. These articles contain several new, novel, and innovative techniques and ideas that may stimulate further research in every branch of pure and applied sciences.

\section{Acknowledgments}

The authors would like to express their deepest gratitude to the reviewers, whose professional comments and valuable suggestions guaranteed the high quality of these selected papers. The editors would like to express their gratitude to the authors for their interesting and novel contributions. They would also like to thank the editorial boards members of this journal, for their support and help throughout the preparation of this special issue. The interested readers are advised to explore these interesting and fascinating fields further. The authors hope that problems discussed and investigated in this issue may inspire and motivate to discover new, innovative, and novel applications in all areas of pure, regional, physical, social, industrial, and engineering sciences.

Khalida Inayat Noor

Muhammad Aslam Noor Syed Tauseef Mohyud-Din

Ahmet Yildirim

Shaher M. Momani 


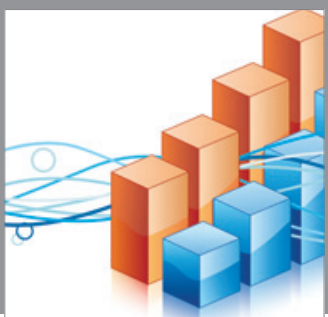

Advances in

Operations Research

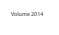

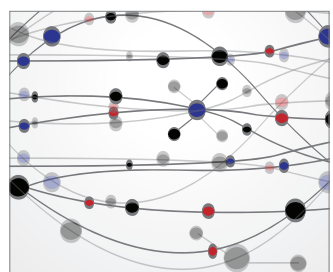

\section{The Scientific} World Journal
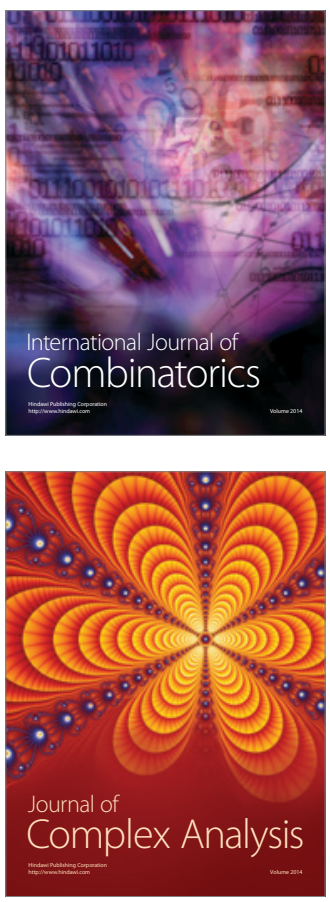

International Journal of

Mathematics and

Mathematical

Sciences
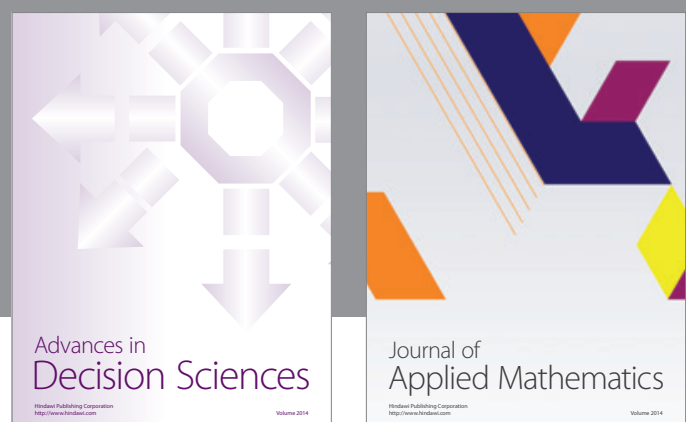

Journal of

Applied Mathematics
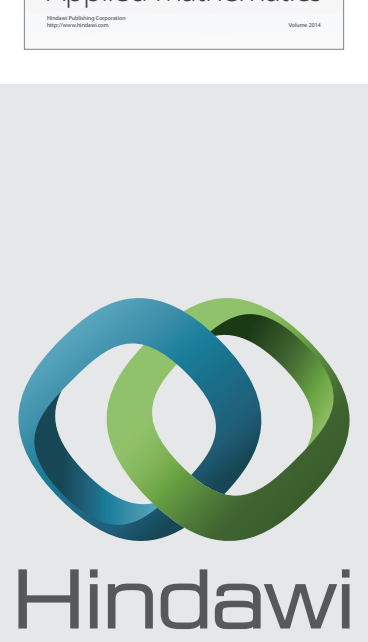

Submit your manuscripts at http://www.hindawi.com
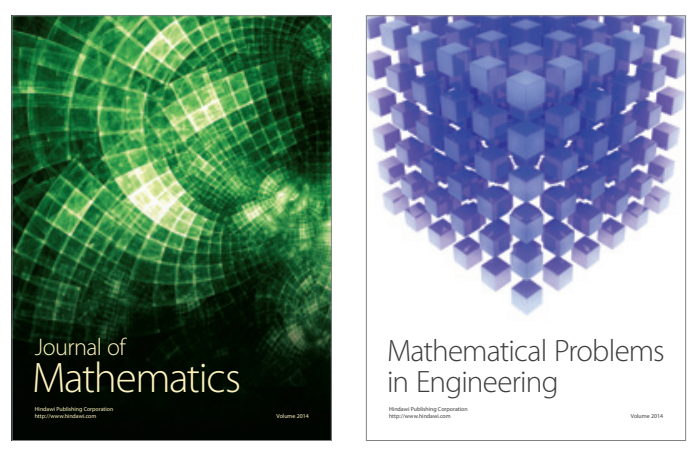

Mathematical Problems in Engineering
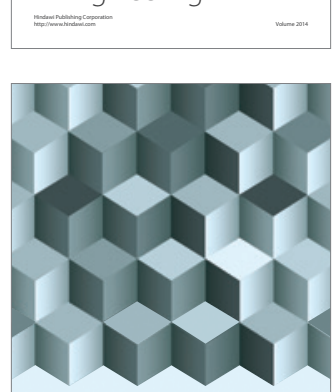

Journal of

Function Spaces
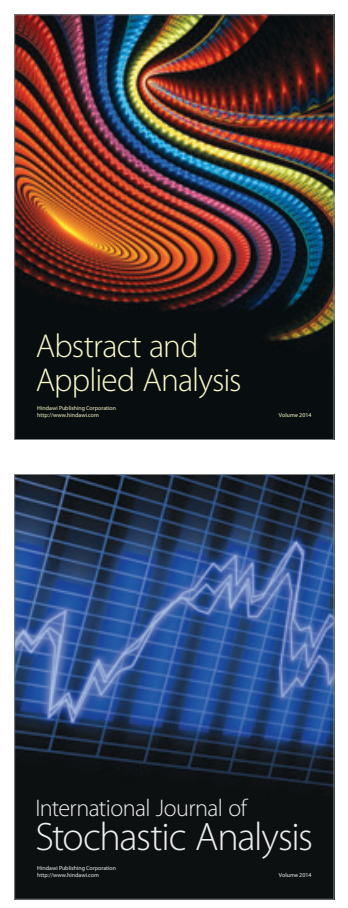

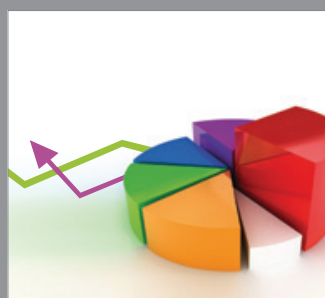

ournal of

Probability and Statistics

Promensencen
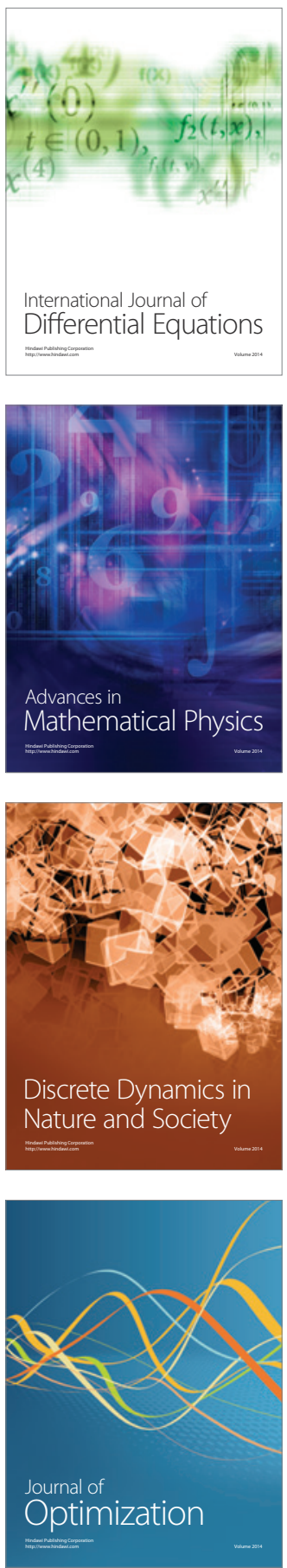